\title{
SARS-CoV-2-related pneumonia can be successfully managed in patients with metastatic neuroendocrine tumors: a critical point of view
}

\author{
F. Spada ${ }^{1} \cdot$ S. Pellicori ${ }^{1} \cdot$ G. Zampino ${ }^{1} \cdot$ L. Funicelli $^{2} \cdot$ L. Gervaso $^{1} \cdot$ A. Laffi ${ }^{1} \cdot$ M. Rubino ${ }^{1} \cdot$ R. Garcia-Carbonero ${ }^{3}$. \\ N. Fazio $\mathbb{1}^{1}$
}

Received: 30 April 2020 / Accepted: 6 July 2020 / Published online: 17 July 2020

(c) Springer Science+Business Media, LLC, part of Springer Nature 2020

\begin{abstract}
Primary neuroendocrine tumors of the thymus are extremely rare. In patients with advanced disease, tumor growth control, and sometimes also syndrome control are the main goals of systemic therapy. Unfortunately, no standard therapies are available in clinical practice; therefore, clinical studies are strongly recommended. Axitinib (AXI) is a tyrosine kinase inhibitor, currently under investigation in an international phase II/III trial including thymic neuroendocrine tumors. Over the past 5 months, the entire world has been facing a devastating medical emergency brought about by a pandemic due to a novel coronavirus known as severe acute respiratory syndrome coronavirus 2 (SARS-CoV-2), which emerged in Wuhan, China, in late 2019. Since then, health professionals have been expending all their efforts on trying to provide the best available treatments for patients involved. Patients with cancer, especially those with thoracic involvement, are at higher risk of coronavirus disease 19 (COVID-19) and its complications because of their immunosuppressive status caused by the cancer and the anticancer therapies. As it remains unclear how to optimally manage such patients, we wished to report our experience with a patient with a metastatic neuroendocrine tumor of the thymus infected with SARS-CoV-2 in the hope that it may provide some insights and reflections on the management of cancer patients during this challenging time in our history.
\end{abstract}

Keywords COVID-19 $\cdot$ NET $\cdot$ Thymus $\cdot$ Neuroendocrine tumors

\section{Introduction}

Thymic (Thy) neuroendocrine tumors (NETs) represent a heterogeneous and rare group of neoplasms including less than $5 \%$ of primary epithelial tumors in the thymus [1]. They can be discovered incidentally or due to symptoms related to tumor mass effect or hormone secretion. In

$\triangle$ N. Fazio

nicola.fazio@ieo.it

1 Division of Gastrointestinal Medical Oncology and Neuroendocrine Tumors, European Institute of Oncology (IEO), IRCCS, Via Ripamonti 435, 20141 Milan, Italy

2 Division of Radiology, European Institute of Oncology (IEO), IRCCS, Via Ripamonti 435, 20141 Milan, Italy

3 Medical Oncology Department, Hospital Universitario Doce de Octubre, Imas12, UCM, Madrid, Spain advanced low/intermediate-grade Thy-NETs [2], prognosis is relatively poor and systemic therapy is usually proposed. Unfortunately, no therapy has been validated so far in clinical practice; thus, treatment within a clinical trial is strongly encouraged.

Axitinib (AXI) is an oral tyrosine kinase inhibitor (TKI) that binds to the kinase domain of receptors 1,2 , and 3 of VEGF, inhibiting intracellular signaling mediated by these receptors and exerting an antiangiogenic action accordingly. Nowadays, it is approved in the treatment of metastatic renal cell carcinoma progressive on sunitinib or other cytokines. AXI, in association with octreotide (OCT) longacting repeatable (LAR) versus placebo plus OCT LAR, is being investigated in NETs within a phase II/III international randomized double-blind study (ClinicalTrials.gov Identifier: NCT01744249), enrolling Thy-NETs as well.

Severe acute respiratory syndrome coronavirus 2 (SARSCoV-2), also known as coronavirus disease 19 (COVID-19), appeared in mainland China, starting from Wuhan, Hubei, in 
late 2019, and resulted in a catastrophic worldwide outbreak [3]. Over the past 5 months, this outbreak has rapidly worsened from an epidemic to a pandemic [4].

In Italy, the first cases were identified at the end of February 2020 and its dramatic spread immediately came afterward [3].

COVID-19 can evolve from a mild respiratory tract illness with cough, sore throat, myalgias, and fever up to severe interstitial pneumonia with severe dyspnea, even proving fatal in many cases [5]. Cancer patients are supposed to be at a higher risk than the general population of contracting the infection and developing complications of COVID-19 due to their baseline and/or anticancer therapyinduced immunosuppressive status. Those with thoracic cancers are particularly at risk $[6,7]$.

\section{A metastatic Thy-NET: a case report}

We report the case of a patient with advanced Thy-NET (atypical carcinoid in accordance with the 2015 WHO classification [2]) on the above-mentioned phase II/III trial with AXI for 1 year with stable disease as best response, alongside pneumonia caused by SARS-CoV-2, successfully treated with medical therapy.

On March 17, 2020, a 73-year-old Italian male patient with Thy-NET with bilateral pleural, mediastinal node, and bone metastases presented for a scheduled consultation with $89 \%$ peripheral oxygen saturation ( $\mathrm{SpO} 2)$, severe asthenia, and exertional dyspnea. The appearance of the aforementioned symptoms, combined with symptoms interpreted as side effects from the antitumoral therapy (mild hypothyroidism and renal failure), supported the suspicion that the patient had been included in the experimental arm. This suspicion had therefore led the treating oncologist to interrupt AXI/Placebo ten days earlier as a precaution.

The patient had previous gastric cancer and concurrent diabetes mellitus on oral metformin. No contacts with suspected cases of COVID-19 were identifiable in his recent clinical history.

Chest CT scan revealed bilateral signs of interstitial pneumonia (Fig. 1a). A throat swab on real-time reverse transcription-polymerase chain reaction was performed, resulting positive for SARS-CoV-2. Laboratory tests showed increased white blood cell counts, neutrophil counts, neuron-specific enolase, and chromogranin-A plasma levels.

In accordance with local procedures, it was recommended that the patient stay isolated at home.

He received hydroxychloroquine $400 \mathrm{mg} /$ day over 5 days and azithromycin $500 \mathrm{mg} /$ day over 14 days. He was phone-monitored by his general practitioner plus the treating oncologist. One week later, PCR and procalcitonin were
$1.7 \times \mathrm{ULN}$ (upper limit of normality) and $48 \times \mathrm{ULN}$, respectively, without further clinical manifestations. Oxygen saturation progressively improved as did symptoms. At 3 weeks from diagnosis, two consecutive throat swabs resulted negative for SARS-CoV-2 and the Chest CT scan showed improvement bilaterally (Fig. 1b). In accordance with some experience reporting a specific role in the management of COVID-19 [8, 9], interleukin-6 (IL-6) and anticardiolipin IgG (CARG) and IgM (CARM) levels were tested and they were found twofold $\times$ ULN and within normal range, respectively. D-dimer was found fivefold $\times$ ULN. As the patient fully recovered, AXI/Placebo were restarted on April 14, 2020 (Fig. 1c).

\section{Discussion}

To the best of our knowledge, this is the first report of a patient with an advanced NET with a COVID-19 pneumonia. Although not receiving any immunosuppressive antitumor therapy, our patient was a high-risk patient due to his advanced thoracic cancer, with pleural and lung parenchymal metastases in addition to his age. Furthermore, he had been heavily pretreated and he had relevant comorbidities, such as diabetes.

At present, no therapy is universally recognized as effective for the COVID-19 pneumonia. At the time of writing, the European Medicines Agency is evaluating over 40 drugs and 12 vaccines for COVID-19. Whether the favorable evolution of COVID-19-related pneumonia in our patient was due to the hydroxychloroquine and antibiotic or to the natural indolent history of the disease is an open question. Moreover, it remains unclear why some people, younger than our patient, or patients without comorbidities have had an aggressive course of COVID-19 infection while others, with all the prerequisites to get worse, as our case, have completely recovered.

How patients with an advanced cancer on chronic antitumor therapy should be managed in order to avoid or treat COVID-19 is a matter of debate.

Some open questions concern the level of risk of COVID-19 for thoracic cancer patients, including patients with NET histology compared with other tumor types; the discontinuation of antitumor therapies when the patient may have mild symptoms of interstitial pneumonia and yet may have an aggressive cancer; and the role of IL-6, CARG/CARM, D-dimer and coagulation in general, as potential predictors of severity of coagulopathy in this frail population.

Some national oncological organizations have published guidance about cancer patient management during the COVID-19 outbreak. Moreover, leading scientific international medical societies, the American Society of Oncology 


\section{$\mathbf{A}$}
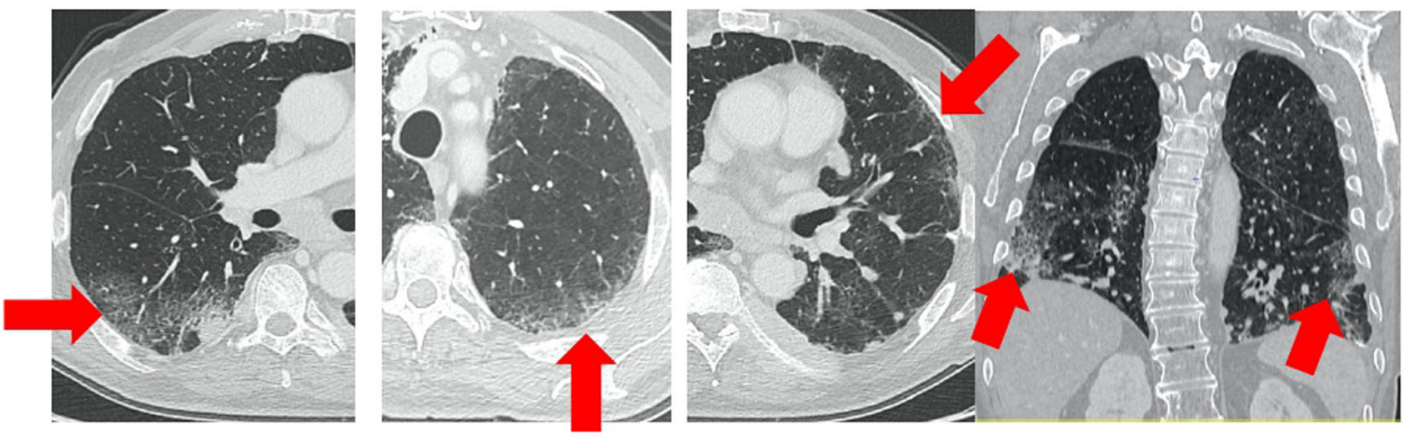

B
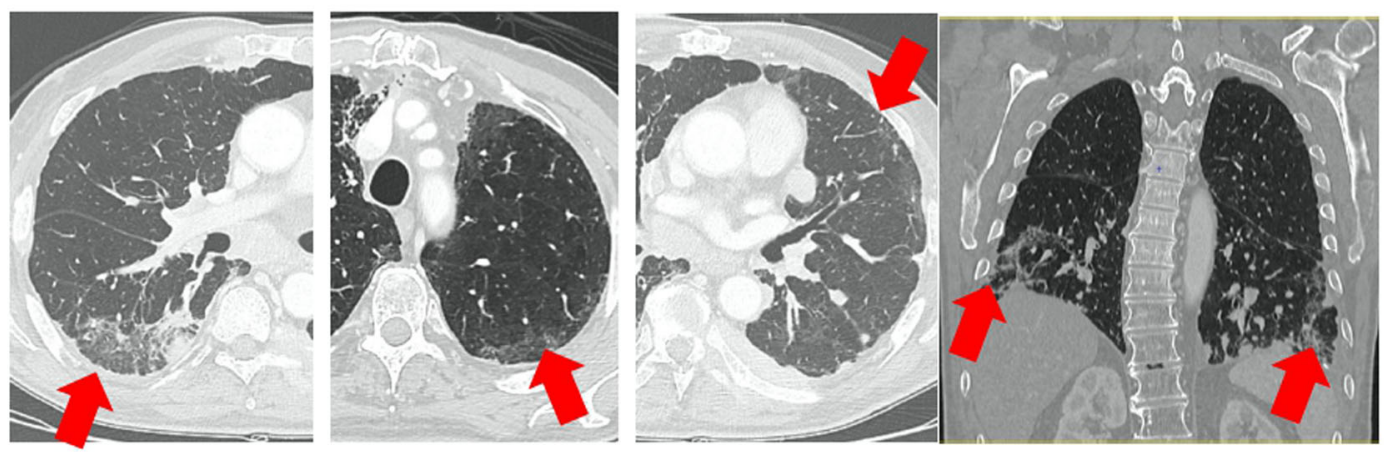

C

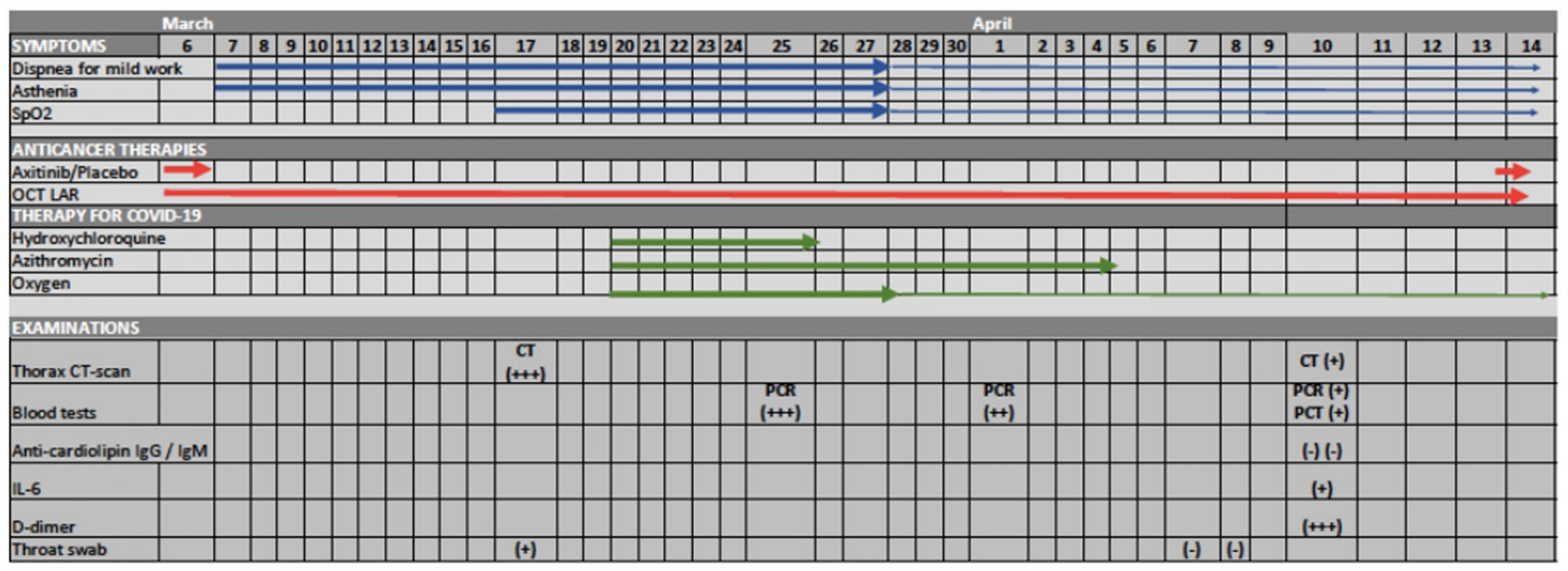

Legend: $\mathrm{SpO2}$ = peripheral oxygen saturation; OCT LAR = octreotide long-acting repeatable; CT = Computed Tomography scan; PCR = Polymerase Chain Reaction; PCT $=$ Procalcitonin; $1 \mathrm{ll}-6=$ interleukin 6

Fig. 1 a Thorax CT scan with radiological signs of interstitial pneumonia from COVID-19 (17 March 2020): subsegmental peripheral patchy ground-glass opacities (GGOs) with obscure boundaries and mainly subpleural distribution associated with some areas of focal thickening. b Thorax CT scan with improved radiological signs of

(ASCO) (http://asco.org) and the European Association of Medical Oncology (http://esmo.org), have now also issued relevant information, frequently asked questions, and advice.

Among them, although the data are limited, ASCO is the only body that strongly suggests interrupting anticancer therapies in patients with active COVID-19, since interstitial pneumonia from COVID-19 (10 April 2020): partial resolution of the ground-glass opacities with septal thickening and reduction of the focal thickening areas. $\mathbf{c}$ Chronological clinical trend. Time course of the clinical features of the patient, therapies, laboratory tests, and CT scan during SARS-CoV-2 pneumonia

continuing treatment may lead to further immunosuppression and risk of serious complications. Accordingly, Zhang et al. [10] reported that cancer patients should avoid therapies that could result in immunosuppression status or have their dosages reduced if COVID-19 coinfection is also present. By contrast, Liang et al. [6] observed that patients with lung cancer did not have a higher probability of serious 
events compared with patients with other cancer types. In this regard, an insightful paper from Mount Sinai Hospital experience showed lower mortality rates of COVID-19 in cancer patients above 50 years of age as compared with patients without cancer [11]. Among cancer patients and SARS-CoV-2 coinfection, they also suggest more intensive surveillance and treatment especially in older patients or those with other comorbidities.

However, no specific recommendations were reported in the aforementioned statements regarding either NETs or targeted therapies.

Zhang et al. [12] illustrated that biological antitumor therapy could be maintained or restarted after a short temporary interruption in patients with thoracic cancer.

Therefore, we suggest that patients with advanced NETs, even thoracic, on chronic TKI and SSAs should not stop their antitumor therapy and be managed on a case-by-case basis when overlapping COVID-19 pneumonia is present.

We are well aware that in our case the specific study design could induce other investigators to think differently. In fact, on the one hand if the patient is included in the conventional arm, the favorable tumor trend could not be influenced by the AXI at all and the tumor stability could depend on the natural history of the tumor itself. On the other hand, if the patient is in the experimental arm, AXI could have a favorable impact on the patient's prognosis indeed its early discontinuation could have a negative effect on the whole therapeutic strategy of the patient. In this latter case, it should be taken into account that the high D-dimer level could underline a potential role of AXI which, based on its specific mechanism of action [13], could favor the occurrence of venous thromboembolic and pulmonary embolic events, as described in a clinical trial [14]. In addition, impaired coagulation parameters have also recently been associated with a higher risk of acute distress respiratory syndrome and death [15] in patients with COVID-19, although the impact of this correlation is yet to be determined. This means that the decision to discontinue AXI in our case should strike a good balance between the goal of tumor control and the potential embolic events.

Moreover, our experience suggests that although cancer patients are associated with the same clinical definition, "oncological", they could be markedly different in terms of immunosuppressive risk and clinical behavior, and even concomitant illness may arise, or already be present. They could thus be managed in personalized ways. Therefore, a generalized approach to cease antitumor therapy in COVID-19 patients in some specific cancer categories could be detrimental. In particular, neuroendocrine neoplasms include not only low-aggressive diseases with a relatively good prognosis despite being metastatic, but also those that are biologically and clinically aggressive. In each case, discontinuing anticancer therapy at an early point could result in undertreatment, adversely affecting the cancer prognosis.

Furthermore, where COVID-19 pneumonia is also present, a case-by-case management is recommended, according to the clinical behavior of the pneumonia, the clinical status of the patient, and status and prognosis of the underlying cancer.

\section{Conclusion}

To conclude, the risk and severity of SARS-CoV-2 may be due to various factors attributable to the patients, the neoplasm and the virus itself. For all these reasons, the decision to pursue antitumoral therapies, especially therapeutic agents without an immunosuppressive effect, should be discussed in a multidisciplinary setting, sharply focusing on the immediate and long-term objectives of the whole therapeutic strategy.

Author contributions All authors contributed to the study conception and design. The first draft of the manuscript was written by F.S. and all authors commented on previous version of the manuscript. All authors read and approved the final manuscript.

\section{Compliance with ethical standards}

Conflict of interest F.S. is the principal investigator of the phase II/III international randomized double-blind study (ClinicalTrials.gov Identifier: NCT01744249) at the IEO (Milan) centre. A.L., M.R., and N.F. are coinvestigators of the phase II/III international randomized double-blind study (ClinicalTrials.gov Identifier: NCT01744249) at the IEO (Milan) centre. R.G.-C. is the global principal investigator of the phase II/III international randomized double-blind study (ClinicalTrials.gov Identifier: NCT01744249). The other authors declare that they have not conflict of interest.

Patient's consent The patient gave the consent to the publication.

Publisher's note Springer Nature remains neutral with regard to jurisdictional claims in published maps and institutional affiliations.

\section{References}

1. P. Strobel, A. Zettl, K. Shilo, W.Y. Chuang, A.G. Nicholson, Y. Matsuno, A. Gal, R.H. Laeng, P. Engel, C. Capella, M. Marino, J. K. Chan, A. Rosenwald, W. Travis, T.J. Franks, D. Ellenberger, I. M. Schaefer, A. Marx, Tumor genetics and survival of thymic neuroendocrine neoplasms: a multi-institutional clinicopathologic study. Genes Chromosomes Cancer 53(9), 738-749 (2014). https://doi.org/10.1002/gcc.22183

2. W.D. Travis, E. Brambilla, A.G. Nicholson, Y. Yatabe, J.H.M. Austin, M.B. Beasley, L.R. Chirieac, S. Dacic, E. Duhig, D.B. Flieder, K. Geisinger, F.R. Hirsch, Y. Ishikawa, K.M. Kerr, M. Noguchi, G. Pelosi, C.A. Powell, M.S. Tsao, I. Wistuba, The 2015 World Health Organization classification of lung tumors: impact of genetic, clinical and radiologic advances since the 2004 classification. J. Thorac. Oncol. 10(9), 1243-1260 (2015). https://doi. org/10.1097/jto.0000000000000630 
3. Report, Coronavirus disease 2019 (COVID-19) situation report70. (WHO, 2020). who.int

4. K.K. Sahu, A.K. Mishra, A. Lal, COVID-2019: update on epidemiology, disease spread and management. Monaldi Arch. Chest Dis. 90(1), (2020). https://doi.org/10.4081/monaldi.2020. 1292

5. W.J. Guan, Z.Y. Ni, Y. Hu, W.H. Liang, C.Q. Ou, J.X. He, L. Liu, H. Shan, C.L. Lei, D.S.C. Hui, B. Du, L.J. Li, G. Zeng, K.Y. Yuen, R.C. Chen, C.L. Tang, T. Wang, P.Y. Chen, J. Xiang, S.Y. Li, J.L. Wang, Z.J. Liang, Y.X. Peng, L. Wei, Y. Liu, Y.H. Hu, P. Peng, J.M. Wang, J.Y. Liu, Z. Chen, G. Li, Z.J. Zheng, S.Q. Qiu, J. Luo, C.J. Ye, S.Y. Zhu, N.S. Zhong, Clinical characteristics of coronavirus disease 2019 in China. N. Engl. J. Med. (2020). https://doi.org/10.1056/NEJMoa2002032

6. W. Liang, W. Guan, R. Chen, W. Wang, J. Li, K. Xu, C. Li, Q. Ai, W. Lu, H. Liang, S. Li, J. He, Cancer patients in SARS-CoV-2 infection: a nationwide analysis in China. Lancet Oncol. 21(3), 335-337 (2020). https://doi.org/10.1016/s1470-2045(20)30096-6

7. L. Bonomi, L. Ghilardi, E. Arnoldi, C.A. Tondini, A.C. Bettini, A rapid fatal evolution of coronavirus disease-19 (COVID-19) in an advanced lung cancer patient with a long time response to nivolumab. J. Thorac. Oncol. (2020). https://doi.org/10.1016/j.jtho. 2020.03.021

8. Y. Zhang, M. Xiao, S. Zhang, P. Xia, W. Cao, W. Jiang, H. Chen, X. Ding, H. Zhao, H. Zhang, C. Wang, J. Zhao, X. Sun, R. Tian, W. Wu, D. Wu, J. Ma, Y. Chen, D. Zhang, J. Xie, X. Yan, X. Zhou, Z. Liu, J. Wang, B. Du, Y. Qin, P. Gao, X. Qin, Y. Xu, W. Zhang, T. Li, F. Zhang, Y. Zhao, Y. Li, S. Zhang, Coagulopathy and antiphospholipid antibodies in patients with Covid-19. N. Engl. J. Med. (2020). https://doi.org/10.1056/NEJMc2007575

9. P. Mehta, D.F. McAuley, M. Brown, E. Sanchez, R.S. Tattersall, J.J. Manson, COVID-19: consider cytokine storm syndromes and immunosuppression. Lancet 395(10229), 1033-1034 (2020). https://doi.org/10.1016/s0140-6736(20)30628-0

10. L. Zhang, F. Zhu, L. Xie, C. Wang, J. Wang, R. Chen, P. Jia, H.Q. Guan, L. Peng, Y. Chen, P. Peng, P. Zhang, Q. Chu, Q. Shen, Y. Wang, S.Y. Xu, J.P. Zhao, M. Zhou, Clinical characteristics of COVID-19-infected cancer patients: a retrospective case study in three hospitals within Wuhan, China. Ann. Oncol. (2020). https:// doi.org/10.1016/j.annonc.2020.03.296

11. H. Miyashita, T. Mikami, N. Chopra, T. Yamada, S. Chernyavsky, D. Rizk, C. Cruz, Do patients with cancer have a poorer prognosis of COVID-19? An experience in New York City. Ann. Oncol. (2020). https://doi.org/10.1016/j.annonc.2020.04.006

12. H. Zhang, C. Xie, Y. Huang, Treatment and outcome of a patient with lung cancer infected with severe acute respiratory syndrome coronavirus-2. J. Thorac. Oncol. 15(5), e63-e64 (2020). https:// doi.org/10.1016/j.jtho.2020.02.025

13. N. Fazio, C.A. Cella, M. Del Re, A. Laffi, M. Rubino, P. Zagami, F. Spada, Pharmacodynamics, clinical findings and approval status of current and emerging tyrosine-kinase inhibitors for pancreatic neuroendocrine tumors. Expert Opin. Drug Metab. Toxicol. 15(12), 993-1004 (2019). https://doi.org/10.1080/ 17425255.2019.1700951

14. T.E. Hutson, V. Lesovoy, S. Al-Shukri, V.P. Stus, O.N. Lipatov, A.H. Bair, B. Rosbrook, C. Chen, S. Kim, N.J. Vogelzang, Axitinib versus sorafenib as first-line therapy in patients with metastatic renal-cell carcinoma: a randomised open-label phase 3 trial. Lancet Oncol. 14(13), 1287-1294 (2013). https://doi.org/10.1016/ s1470-2045(13)70465-0

15. N. Tang, D. Li, X. Wang, Z. Sun, Abnormal coagulation parameters are associated with poor prognosis in patients with novel coronavirus pneumonia. J. Thromb. Haemost. 18(4), 844-847 (2020). https://doi.org/10.1111/jth.14768 